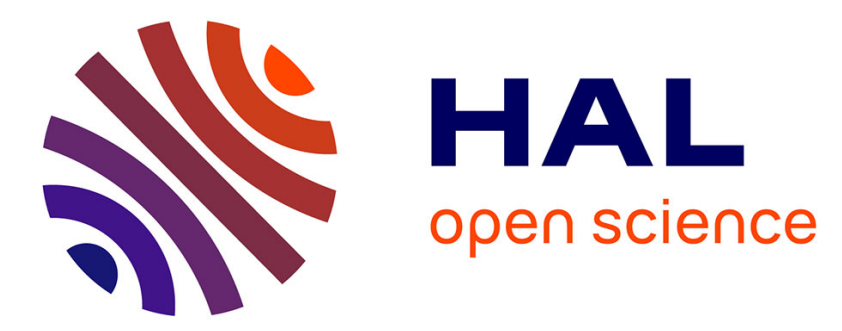

\title{
Conducted EMI prediction for integrated class D audio amplifier
}

\author{
Roberto Mrad, Florent Morel, Gaël Pillonnet, Christian Vollaire, Angelo
}

Nagari

\section{- To cite this version:}

Roberto Mrad, Florent Morel, Gaël Pillonnet, Christian Vollaire, Angelo Nagari. Conducted EMI prediction for integrated class D audio amplifier. 18th IEEE ICECS, 2011, Beyrouth, Lebanon. pp.4, 10.1109/ICECS.2011.6122295 . hal-01103646

\section{HAL Id: hal-01103646 \\ https://hal.science/hal-01103646}

Submitted on 15 Jan 2015

HAL is a multi-disciplinary open access archive for the deposit and dissemination of scientific research documents, whether they are published or not. The documents may come from teaching and research institutions in France or abroad, or from public or private research centers.
L'archive ouverte pluridisciplinaire HAL, est destinée au dépôt et à la diffusion de documents scientifiques de niveau recherche, publiés ou non, émanant des établissements d'enseignement et de recherche français ou étrangers, des laboratoires publics ou privés. 


\title{
Conducted EMI Prediction for Integrated Class D Audio Amplifier
}

\author{
Roberto Mrad $^{123}$, Florent Morel $^{1}$, Gaël Pillonnet ${ }^{2}$, Christian Vollaire $^{1}$, Angelo Nagari ${ }^{3}$ \\ ${ }^{1}$ University of Lyon, ECL \\ Ampère, UMR CNRS 5005 \\ 36, avenue Guy de Collongue \\ 69134 Ecully cedex - France \\ firstname.lastname@ec-lyon.fr
}

\begin{abstract}
The aim of this paper is to predict the delivered currents at the output of an integrated audio switching amplifier for EMI prediction. Impedance matrices are used to model the different passive parts of the system. Hereafter, all the matrices are associated in a single one, where the resulting matrix and the output voltages in open circuit are used to predict the output currents spectra directly in the frequency domain. This method can be used by system designers and system integrators in order to study their systems EM emissions before assembling the different parts of the system. The experimental application of this method gives good accuracy up to $10 \mathrm{MHz}$ (twenty times the switching frequency).
\end{abstract}

Keywords-Integrated Class D audio amplifier; EMI prediction; Impedance matrix; Conducted EMI

\section{INTRODUCTION}

Over the years, cell phones have become more and more complicated. Expert designers are introducing complex systems for more sophisticated functionalities. These are composed of calculation and power management circuits, fabricated in submicron technologies, calculating with extremely high speed (processors, controllers etc.), switching at high frequencies (DC-DC convertors) and mounted on a small area of a portable cellphone. This trend for size reduction also reduces the supply voltage which leads to a better power efficiency. However, this smaller supply voltage reduces the noise margin which increases the system susceptibility to electromagnetic interference (EMI). As higher switching frequencies lead to much conducted and radiated noise emissions, the ElectroMagnetic Compatibility (EMC) can no longer be neglected.

In the case of the audio systems, integrated class D amplifiers (Fig.1) show good agreement in terms of the power efficiency and audio quality [1]. However, the switching nature of these amplifiers (the switching frequency is between $300 \mathrm{KHz}$ and $2 \mathrm{MHz}$ ) generates many harmonics that can disturb the surrounding electronics such as processors and RF components. For that reason, designers use some integrated design techniques [2-5] to reduce the ElectroMagnetic Interference (EMI). Nevertheless, in most of the cases these improvements are not sufficient, and an EMI filter must be inserted before the load to prevent the conducted emissions from propagating through the tracks, especially between the amplifier and the speaker.

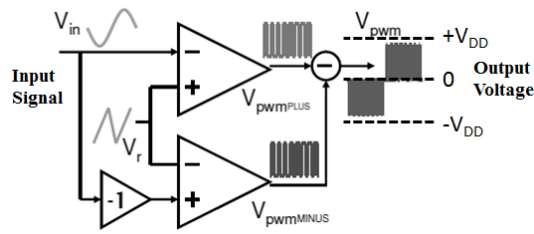

(a): PWM ternary modulation technique

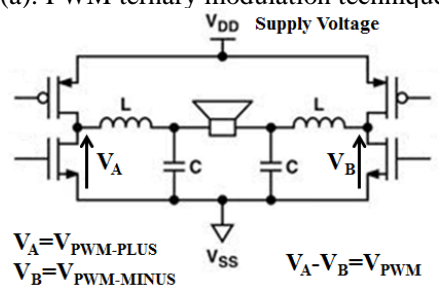

(b): Class D power stage

Figure 1: Differential Class D audio amplifier

To study the EMIs, experts now study the already built systems and try to generate an equivalent EMI model [6]. Indeed, a post-construction model can be useful, but it is not able to predict the frequency spectrum in order to solve the EMI problems before construction, which mean time to market and costs cannot be reduced. Designers therefore need an EMI predictive method to solve their problems at the early design stage, with good accuracy at high frequencies and with a short simulation time. In order to do that, the present method allows system integrators to use the already built parts of the system like integrated circuits (e.g. switching class D amplifiers), filters, speakers etc., and predict the behavior of their association. For example, if several filters and loads were modeled, the system integrator could determine which filter and load combination shows the best electromagnetic efficiency with a given power amplifier.

In this paper, the method presented in [7] is used to model the output passive parts of integrated Class D switching amplifier with impedance matrices. Afterwards, the output voltages are measured in open circuit to predict the current frequency spectra thanks to the already determined impedance matrix. 
This paper is presented as follows: in section II shows the passive parts modeling by impedance matrices. Section III uses the impedance matrix determined in section II to predict the currents spectra.

\section{PASSIVE PARTS MODELLING}

\section{A. Five terminal passive blocks modelling}

The approach presented in [7] consists in decomposing a system with two active conductors and a ground plane in blocks. These blocks can then be associated in order to rebuild the behavior of the system as a single block. For example, Fig.2.a shows the decomposition of a cellphone audio amplification system into blocks.

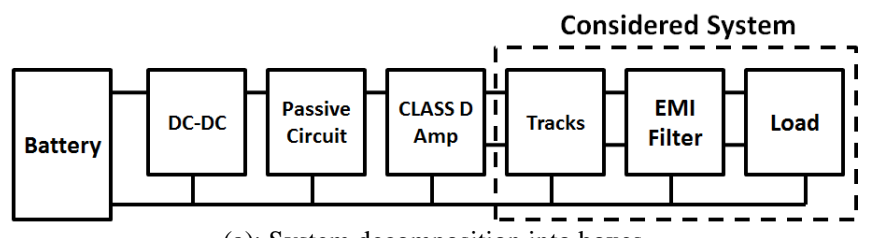

(a): System decomposition into boxes

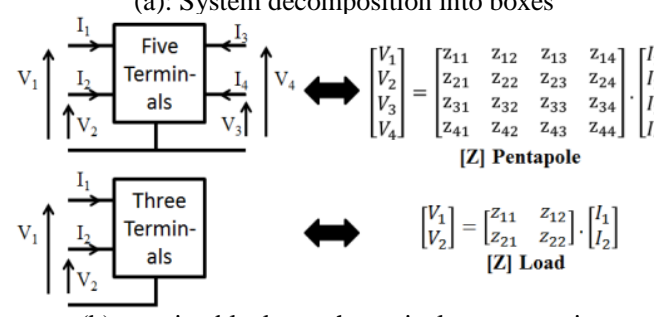

(b): passive blocks mathematical representation

Figure 2: Block decomposition of an amplification system

In fact in this figure, there are two types of blocks. The first represents the active blocks (Class-D amplifier and the DC-DC converter); the second, represents the passive blocks (filters, tracks, loads etc).

Since the class D amplifier has an H-bridge power stage and a differential output (Fig.1), each passive output block is considered as five-terminal-box, because it has two voltages and two currents on the input as well as on the output, except the load (loudspeaker in this case). In fact, the load output cannot be electrically measured so the load is modeled as a three-terminal-block with only two voltages and two currents on its input. Then, each five-terminal-block block is mathematically represented by a $4 \times 4$ impedance matrix (Fig.2.b), and the three-terminal-block (load) is represented by a $2 \times 2$ impedance matrix as shown in Fig.2.b. These impedance matrices can be measured for post-construction modeling (impedance meter, vector analyzer etc.), or simulated for preconstruction modeling [7].

\section{B. Block association}

Once the matrices of the different blocks in the considered sub-system are determined, they can be associated to rebuild the sub-system into a simple block modeled by a $2 \times 2$ impedance matrix (Fig.3). This resulting matrix contains the behavior of the whole sub-system including the defects of all the blocks. It also contains the impedances of the common and differential mode, as well as the mode conversion.

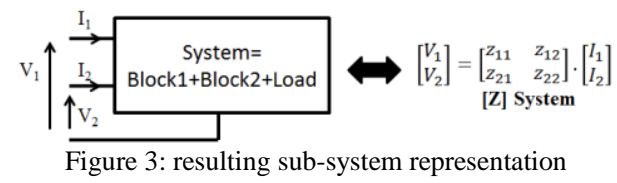

\section{Experimantal application}

Two EMI filters and a mobile phone speaker are considered for validation (Fig.4). The first filter (Filter 1) is recommended with the evaluation kit of an existing Class-D amplifier [8]. The second filter (Filter 2) is similar to Filter 1 but with other capacitances values. The load is a micro-speaker used in cellphones applications [9].

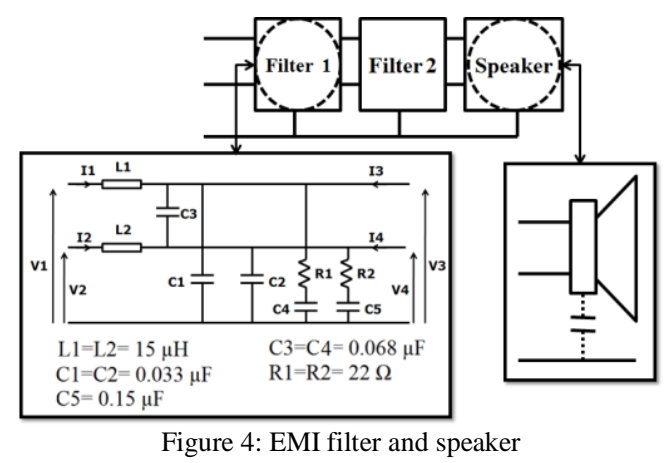

Using the Agilent 4294A impedance analyzer [10], the impedance matrix elements of Filter 1 and Filter 2 are measured. Hereafter, the matrices are mathematically associated to get a single $4 \times 4$ impedance matrix for both blocks. After that, the resulting matrix of Filter 1 and Filter 2 is associated with the speaker impedance matrix. At the end of this procedure, a single $2 \times 2$ impedance matrix is obtained. This matrix contains the behavior of the entire associated blocks (Filter 1, Filter 2 and the Speaker).

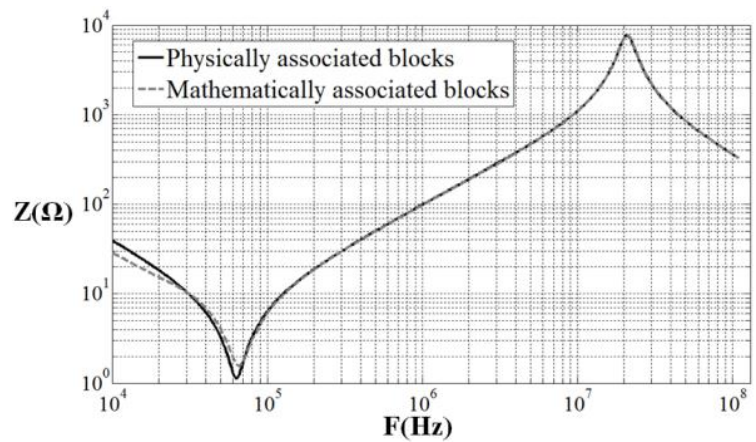

(a): $Z_{\mathrm{R} 11}$

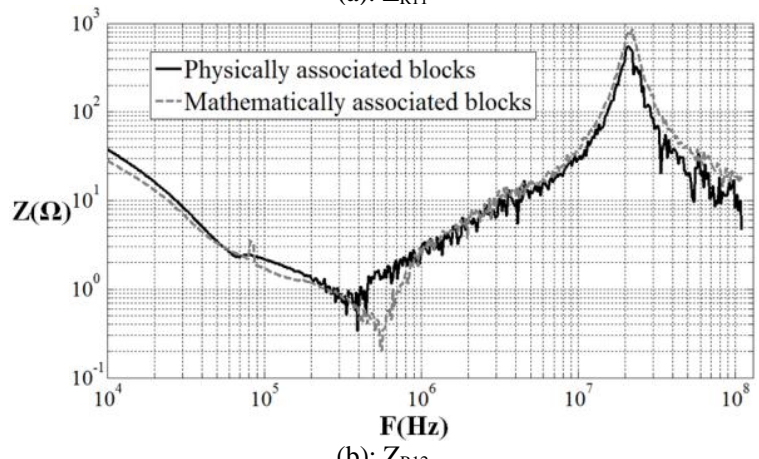

Figure 5: Matrix elements from the Filter 1- Filter 2 - Speaker association 
Fig.5.a shows that the calculated and measured $Z_{R 11}$ superimpose. In Fig.5.b, despite the small difference between the measured and the calculated $Z_{R I 2}$ at some frequencies, these block associations can be considered as valid. Therefore, the resulting matrix of the output passive circuit will be used in the next section for current frequency spectrum prediction.

\section{CURRENT PREDICTION}

A differential sinusoidal audio signal is applied on the inputs of the class-D amplifier (Fig.7). The output voltages of the class-D audio amplifier are measured at the same time, using the Tektronix DPO2014 digital oscilloscope [11] when the output passive circuit (Filter 1 - Filter 2 - Speaker) is disconnected from the amplifier. It is important to note that the voltages are measured in the time domain, and not directly in the frequency domain, because the phase between the two voltages is needed for currents calculation. Hereafter, the FFT is applied to the measured voltages to obtain the frequency spectra. Finally, voltages spectra are multiplied with the inverted impedance matrix to predict the currents frequency spectra. For validation, the currents are also measured with the same digital oscilloscope and a P6022 AC current probe [12] as shown in Fig.6 (where currents are measured with the output passive circuit connected). The measured current spectrum and the calculated current spectrum are both plotted in Fig.8 in order to easily compare them.

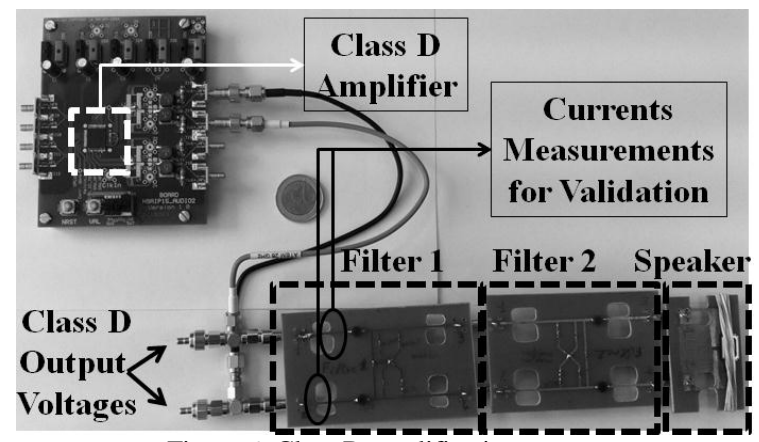

Figure 6: Class D amplification system

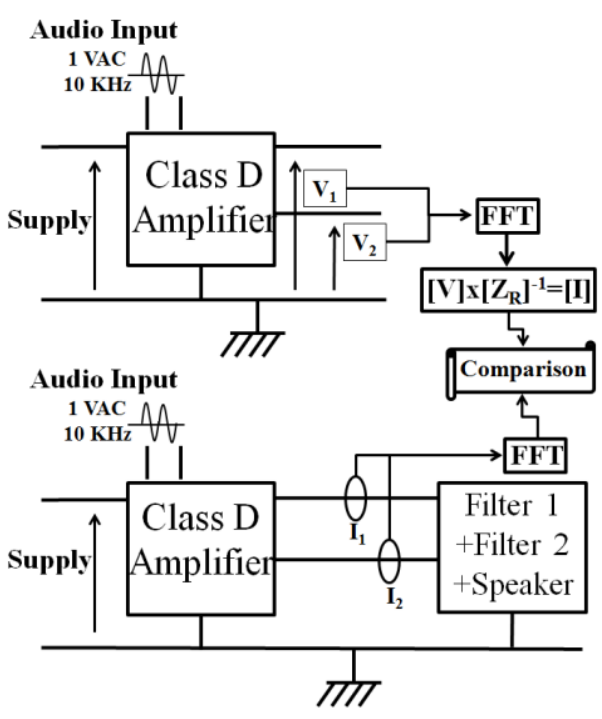

Figure 7: Current prediction validation method

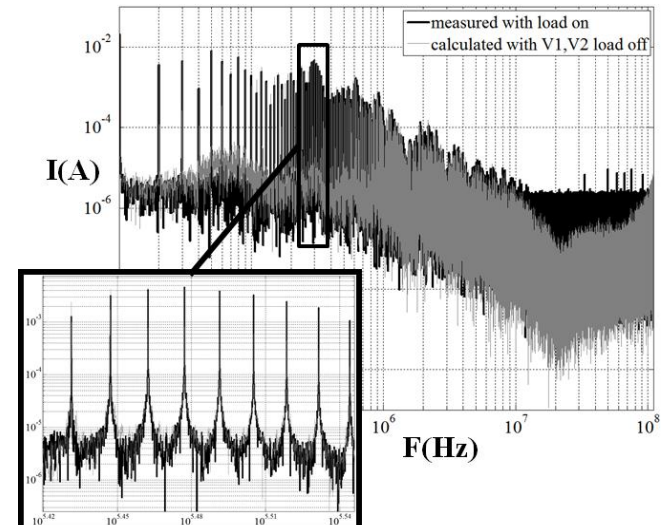

Figure 8: Measured and calculated current spectrum comparison

As can be seen in Fig. 8 the calculated and the measured spectra superimpose up to $10 \mathrm{MHz}$. The current spectrum prediction is valid up to $10 \mathrm{MHz}$, and the resulting matrix perfectly reflects the behavior of the output passive circuit.

Above $10 \mathrm{MHz}$, the spectrum level of the measured current remains the same; this is certainly the noise floor of the measurement equipment. Indeed, the Tektronix DPO2014 digital oscilloscope has only 8 bits for quantization. To confirm this, another measurement was made with a current probe disconnected from the circuit. The results in Fig.9 show that the measured current retains the noise floor level above $10 \mathrm{MHz}$ which thus explains the difference between the measured and the predicted current spectra in Fig.8. To see this clearly, Fig.10 shows a zoom on the measured time domain current.

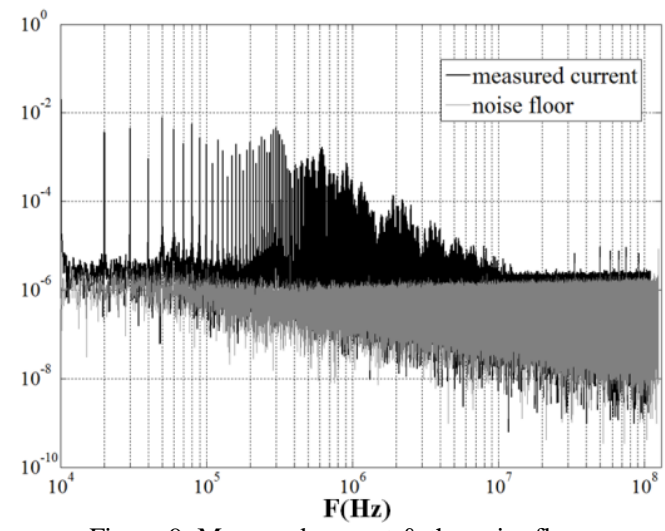

Figure 9: Measured current \& the noise floor

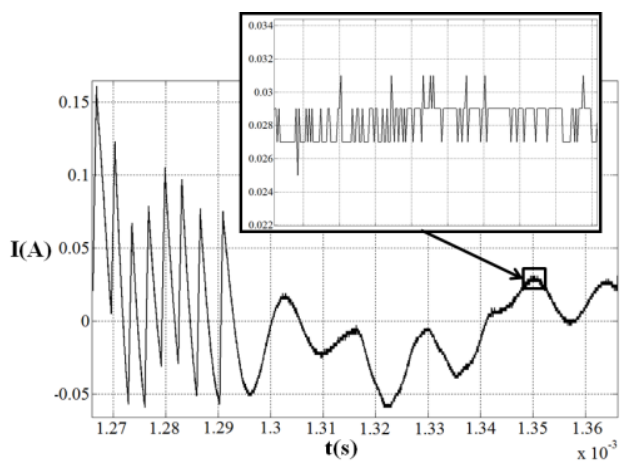

Figure 10: Time domain current signal 
Here we can see that the signal moves by a fixed amount between fixed amplitude values which reveal the existence of a constant noise floor for the equipment used. Therefore, the analog to digital conversion thus introduces quantization noise frequencies.

To overcome this problem, two possible solutions can be considered. In the first hand the output voltage can be measured in two steps, which means the low frequencies are measured with a low pass filter, and the high frequencies are measured with a high pass filter. Then the resulting spectra are merged in a single one. In the other hand the output voltage can be measured using 12 bits oscilloscope, where the digital quantization error is reduced.

In this paper the second solution was taken as an option. The Lecroy WaveRunner $6 \mathrm{Zi}$ digital oscilloscope [13] is used in the same test bench to measure the Class D amplifier output voltages in the same configuration but this time with twice of the sampling frequency and with 12 bits of digital quantization. The results are drawn in Fig.11.

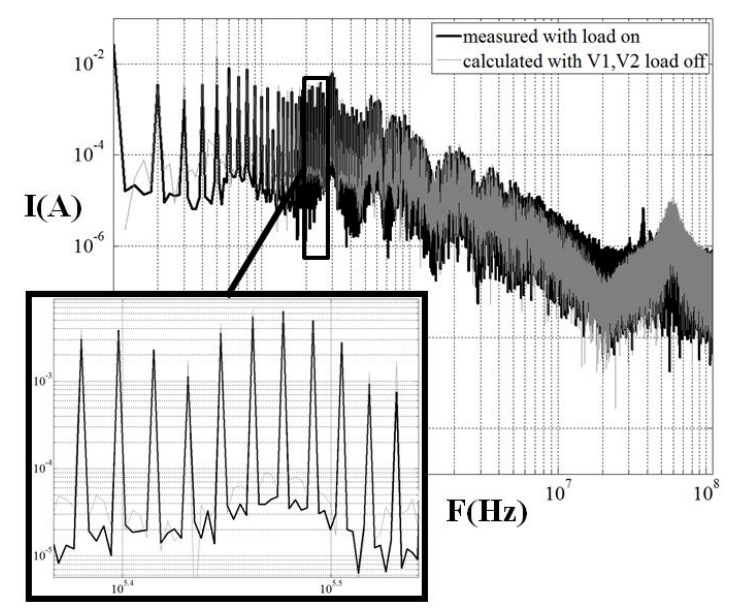

Figure 11: Measured and calculated current spectrum comparison

Fig.11 shows that the measured and the calculated current also superimpose up to $10 \mathrm{MHz}$. Moreover the $12 \mathrm{bits}$ oscilloscope used allowed reducing the noise floor for the measured signal above $10 \mathrm{MHz}$. However Fig. 11 shows also that this method is still not validated above $10 \mathrm{MHz}$. This difference at high frequency between the calculated and the measured current might be the nonlinearity of the passive components in the filters. The inductance and capacitance values are not similar when switching a high current level or when measuring with sinusoidal low level current (impedance analyzer measurements). For that, in future work the passive blocks impedances will be measured around a polarized DC voltage to test the importance of this phenomenon.

\section{CONCLUSION}

The present approach uses the impedance matrices to predict and study the EMIs before assembling the pre-constructed parts of the system. The electronic systems are decomposed into blocks. Each passive part of the system is modeled by an impedance matrix which includes all its imperfections. Hereafter these matrixes can be then cascaded in order to rebuild the system in simple $2 \times 2$ matrix. Furthermore, using the resulting matrix and the open-circuit voltages, the flowing current frequency spectrum can be predicted. This method allows system integrators to isolate the different parts of the system into blocks, and then explore different placement and parts combinations for better electromagnetic compatibility. The block association has been validated up to $110 \mathrm{MHz}$, and for the moment, the current prediction is validated up to $10 \mathrm{MHz}$. It is important to note that in this paper the opencircuit voltages of the integrated Class D audio amplifier are used for current prediction, but it cannot be applied for any switching circuit (especially those including current closedloop controls). The future work will be focused on the current prediction for higher frequencies, and predicting the current and voltages spectrum in different places in the system.

\section{REFERENCES}

[1] Marko Berkhout, "Audio Class D Amplifiers in Mobile application", IEEE International Symposium on Circuit and Systems, pp. 1169-1172, May 2009.

[2] G. Pillonnet, R. Cellier, N. Abouchi, A. Nagari, "A High Performance Switching Audio Amplifier Using Sliding Mode Control”, IEEE Circuits and Systems and TAISA conference, pp. 305-309, 2008.

[3] G. Pillonnet, R. Cellier, N. Abouchi, A. Nagari, "A Topological Comparision of PWM and Hysterisis Control in Switching Audio Amplifier", IEEE Asia Pacific Conference on Circuits and Systems APCCAS, pp.668-671, Dec. 2008.

[4] C.F. Edwards "Efficient switching output stages methods" U.S. Patent 7190225, 13 Mar 2007.

[5] Xin Ming, Zao Chen, “An Advanced Spread Spectrum Architecture Using Pseudorandom Modulation to Improve EMI in Class D Amplifier" Power Electronics, IEEE Transactions. August 2010.

[6] M. Foissac, JL. Schanen, G. Frantz, D. Frey, C. Vollaire, "System Simulation for EMC Network Analysis" Applied Power Electronics Conference and Exposition (APEC), 2011 Twenty-Sixth Annual IEEE, pp. 457, March 2011.

[7] R. Mrad, F. Morel, G. Pillonet, C. Vollaire, D. Labrousse, "Differential Passive Circuit Modeling with Pentapolar Impedance Matrices", EMC Europe 2011.

[8] MAXIM 9700B datasheet http://www.maxim-ic.com/datasheet/ index.mvp/id/4275 [Revised May 24, 2011].

[9] Micro Speaker http://www.seltech-international.com/index.php?/ en/Products/NXP/Speakers/NXP-Speakers/65-RA9.6x13.6x2.9.html [Revised May 24, 2011].

[10] Agilent 4294A Precision Impedance Analyzer datasheet, http://www.home.agilent.com/agilent/facet.jspx?cc=FR\&lc=fre \&k=4294 A\&sm=g [Revised May 24, 2011].

[11] Tektronix DPO 2014 Digital oscilloscope datasheet http://www.tek.com/ products/oscilloscopes/mso2000/ [Revised May 24, 2011].

[12] Tektronix P6022 AC Current Probe datasheet http://www2.tek.com/ cmswpt/psdetails.lotr?ct=PS\&ci=13491\&cs=psu\&lc=EN [Revised May 24, 2011].

[13] Lecroy WaveRunner $6 \quad \mathrm{Zi}$ Digital oscilloscope datasheet http://www.lecroy.com/oscilloscope/oscilloscopeseries.aspx?mseries=35 2\&capid=102\&mid=504 [Revised May 24, 2011]. 